\title{
Paeonol induces antitumor effects in hepatocellular carcinoma cells through survivin via the cyclooxygenase-2/prostaglandin E2 signaling pathway
}

\author{
Hao Liu" ${ }^{\#}$, Congjun Zhang" \\ Department of Oncology, The First Affiliated Hospital of Anhui Medical University, Hefei, China \\ Contributions: (I) Conception and design: C Zhang; (II) Administrative support: H Liu; (III) Provision of study materials or patients: C Zhang; (IV) \\ Collection and assembly of data: C Zhang; (V) Data analysis and interpretation: H Liu; (VI) Manuscript writing: All authors; (VII) Final approval of \\ manuscript: All authors. \\ \#These authors contribute equally to this work. \\ Correspondence to: Congjun Zhang. Department of Oncology, The First Affiliated Hospital of Anhui Medical University, 218 Jixi Street, Hefei 230000 , \\ China. Email: zcj228zj@126.com.
}

Background: Hepatocellular carcinoma (HCC) is one of the most life-threatening malignancies worldwide
due to the lack of significant improvement in therapeutic methods. This study aimed to unravel the effects of
paeonol, the main active component of Paeonia suffruticosa, on survivin, a key molecule in tumorigenesis, and
elucidate the mechanisms by which paeonol exerts antineoplastic effects in human HCC cells.
Methods: Immunohistochemistry (IHC) was used to study the expression levels of survivin and
cyclooxygenase-2 (COX-2) in 57 human HCC tissue samples. Human HCC cell lines (HepG2 and SMMC-
7721 ) were treated with prostaglandin E2 (PGE2). Subsequently, the cells were treated with paeonol and
NS-398, and the expression levels of survivin, COX-2, and PGE2 were evaluated by Western blotting and
enzyme-linked immunosorbent assay (ELISA), respectively. Fluorescence-activated cell sorting (FACS) and
terminal deoxynucleotidyl transferase dUTP nick end labeling (TUNEL) staining were performed to analyze
cell proliferation and apoptosis. Results: Survivin was expressed in 47 of 57 human HCC tissue samples, as observed by IHC, and its expression was correlated with COX-2 activity. Furthermore, Western blotting showed that the expression of survivin was increased in HepG2 and SMMC-7721 cells treated with PGE2, the biosynthesis of which is mainly regulated by COX-2. Interestingly, FACS and TUNEL staining demonstrated that paeonol significantly inhibited the proliferation of HepG2 and SMMC-7721 cells and induced apoptosis, concomitant with the downregulation of survivin. The levels of COX-2 and PGE2 were also reduced by paeonol, as confirmed by Western blotting and ELISA, respectively. To determine the mechanism by which paeonol inhibited survivin in HCC cells the effects of COX-2 expression on surviving were studied. Treatment with the COX-2 selective inhibitor NS398 effectively decreased the levels of PGE2 and survivin, inducing apoptosis in a manner similar to that of paeonol. Survivin expression was increased by PGE2 treatment but was blocked by paeonol, which suggests that paeonol inhibits survivin by inhibiting the COX-2/PGE2 signaling pathway.

Conclusions: To the best of our knowledge, this is the first study to demonstrate that paeonol can exert antitumor effects on HCC cells by targeting survivin via the COX-2/PGE2 signaling pathway. Paeonol could therefore be considered as a potential therapeutic candidate for HCC.

Keywords: Cyclooxygenase-2 (COX-2); hepatocellular carcinoma (HCC); paeonol; prostaglandin E2 (PGE2); survivin

^ ORCID: 0000-0002-1281-0567. 
Submitted Jan 08, 2020. Accepted for publication Sep 17, 2020.

doi: $10.21037 /$ tcr-20-322A

View this article at: http://dx.doi.org/10.21037/tcr-20-322A

\section{Introduction}

Hepatocellular carcinoma (HCC) accounts for more than $80 \%$ of primary liver cancer cases and is the fourth leading global cause of cancer-related mortality (1). It is estimated that about $85 \%$ of HCC cases occur in eastern Asia and sub-Saharan Africa (2). The treatment for late-stage patients who are no longer eligible for local treatment such as curative hepatic resection, tumor ablation, or transarterial therapy, is systematic therapy, including molecular targeted therapy and immune checkpoint therapy (3). Previous studies have focused on angiogenesis as a therapeutic target; the multikinase inhibitors sorafenib and lenvatinib have been approved as first-line targeted therapy, while the multitarget tyrosine inhibitors regorafenib and cabozantinib have been approved as second-line therapy. Meanwhile, immune checkpoint therapy is still under investigation (4). However, survival is still limited for HCC patients, with the 5 -year survival rate currently standing at a relatively low $18 \%$ (5). Therefore, identifying new therapeutic targets for HCC is vital.

Paeonol (2'-hydroxy-4'-methoxyacetophenone) (Figure 1), which is derived from the root cortex of Paeonia suffruticosa, exhibits various pharmacological properties and has been approved for clinical use by the National Medical Products Administration of China. Because of its antiinflammatory and immunoregulatory effects, it is mostly used in the treatment of inflammatory diseases (6). Moreover, paeonol has been reported to exert antitumor activities in ovarian cancer cells (7), prostate cancer cells (8), breast cancer cells (9), and gastric cancer cells (10). Previous studies have shown that paeonol induces apoptosis of hepatoma cells via the nuclear factor kappa-B (NF- $\kappa \mathrm{B})$ pathway (11). Paeonol has also been shown to decrease oxidative injury and improve immunity in rats with HCC (12). In our previous study, paeonol reversed chemoresistance to doxorubicin in HCC cells via a COX-2 mediated pathway (13); however, further investigation to fully understand the mechanism of action of paeonol in HCC is needed.

Survivin, which is encoded by the BIRC5 gene, is a member of the inhibitor-of-apoptosis protein family and is constitutively expressed in embryonic and fetal tissues but not in adult tissues. Further, survivin is overexpressed in multiple cancer types, including colorectal carcinoma (14), breast cancer (15), and lung cancer (16). Previous studies have revealed that survivin promotes tumorigenesis by interfering with mitosis, apoptosis, and autophagy, as well as with antitumor immune responses (17). Despite the attention that survivin has received as a possible therapeutic target in malignant diseases, the correlation of survivin with disease prognosis and the mechanisms by which it drives tumorigenesis are not fully understood (18). Elucidation of the mechanisms of survivin regulation could, therefore, facilitate the identification of new potent anticancer drugs for HCC patients.

The antitumor effects exerted by paeonol via survivin regulation in HCC have yet to be determined. A previous study reported that another Chinese traditional medicine, sinomenine, repressed COX-2, which is an inducible enzyme of prostaglandin synthesis with prostaglandin E2 (PGE2) as a downstream product (19), and survivin expression while inhibiting cell proliferation and inducing apoptosis in esophageal carcinoma (20). Our previous study showed that paeonol could decrease the levels of COX-2 induced by tunicamycin in HCC cells (13). Thus, we hypothesized a correlation between survivin, COX2 , and paeonol, and the present study aimed to investigate the effects of paeonol on survivin via the COX-2/PGE2 pathway in HCC cells. Immunohistochemistry (IHC) was used to study the expression of survivin and COX2. The expression of survivin and COX-2 and that of PGE2 in human HCC cells (HepG2 and SMMC-7721) treated with PGE2, and subsequently, with paeonol and NS-398 were evaluated by Western blotting and enzymelinked immunosorbent assay (ELISA), respectively. Cell proliferation and apoptosis were studied using fluorescenceactivated cell sorting (FACS) and terminal deoxynucleotidyl transferase dUTP nick end labeling staining (TUNEL) assay.

The findings of this study potentially prove the candidature of paeonol as an alternate therapeutic option for HCC patients.

The authors are accountable for all aspects of the work, ensuring that questions related to the accuracy or integrity 


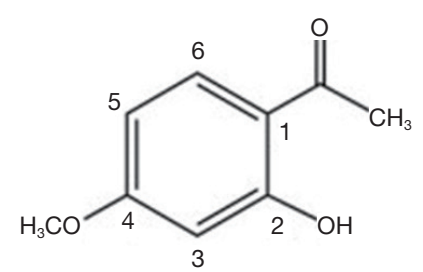

Figure 1 Molecular structure of paeonol.

of any part of the work were appropriately investigated and resolved.

We present the following article in accordance with the MDAR checklist (available at http://dx.doi.org/10.21037/ tcr-20-322A).

\section{Methods}

The study was conducted in accordance with the Declaration of Helsinki (as revised in 2013). The protocol was approved by the institutional review board of Anhui Medical University (approval no. Quick-PJ 2019-14-21), and informed consent was given by all patients enrolled.

\section{HCC specimens}

Paraffin-embedded cancerous and noncancerous adjacent tissues were collected from 57 HCC patients [49 male, 8 female; median age, 50.7 (range, 18-84) years] who underwent surgery in the First Affiliated Hospital of Anhui Medical University between 2001 and 2007. Patients who received neoadjuvant chemotherapy, radiation, or transcatheter arterial chemoembolization before surgery were excluded. All specimens were pathologically confirmed by two expert pathologists according to the World Health Organization classification (21).

\section{Immunobistochemical analysis}

Paraffin-embedded tissue sections, 5 - $\mu$ m thick were deparaffinized in xylene, followed by rehydration in graded alcohol and 3\% hydrogen peroxide in methanol. Then, $0.01 \mathrm{M}$ sodium citrate buffer ( $\mathrm{pH}$ 6.0) was added and the sections were heated in a microwave oven at 360-600 W for $10 \mathrm{~min}$ for antigen retrieval. The sections were then incubated with primary goat survivin and COX-2 antibodies (Santa Cruz Biotechnology, Santa Cruz, CA, USA), both diluted 1:100 for 1 hour, followed by overnight incubation with biotinylated anti-goat secondary antibody diluted 1:100 (Santa Cruz Biotechnology). Next, peroxidaseconjugated streptavidin was added to the samples, followed by diaminobenzidine solution and hematoxylin. Finally, a drop of mounting medium (Thermo Fisher) was added to the slides and the slides were observed under a microscope (Leica, Wetzlar, Germany). Samples showing staining in $>10 \%$ of the tumor cells were considered positive.

\section{Cell culture and treatment}

The HepG2 and SMMC-7721 human HCC cell lines (Shanghai Cell Bank, Chinese Academy of Sciences, Shanghai, China) were used in this study. HepG2 and SMMC-7721 cells were grown in Dulbecco's Modified Eagle Medium (Gibco BRL Life Technologies, Grand Island, NY, USA) with $10 \%$ fetal bovine serum, $100 \mathrm{U} / \mathrm{mL}$ penicillin, and $100 \mu \mathrm{g} / \mathrm{mL}$ streptomycin in a humidified incubator with $5 \% \mathrm{CO}_{2}$ at $37^{\circ} \mathrm{C}$. The cells were treated as previously reported with $10 \mu$ M PGE2 (Sigma Chemical, St. Louis, MO, USA) for 6, 12, and 24 hours (22), with paeonol $(10 \mathrm{mg} / \mathrm{mL}$, First Pharmaceutical Factory of Shanghai, Shanghai, China) (47, 94, 188, and $376 \mu \mathrm{M})$ for 24 and 48 hours (23), and with the COX-2 inhibitor NS-398 $(50 \mu M)$ (Sigma Chemical) for 24 hours (24).

\section{MTT assay}

HepG2 cells and SMMC-7721 cells were seeded in 96well plates at $1 \times 10^{4}$ cells/well. Different concentrations of paeonol $(47,94,188$, and $376 \mu \mathrm{M})$ were added to the cells, and after 24 and 48 hours, cell viability was assessed using a previously described method for MTT assay (13). The cells were then incubated with $20 \mu \mathrm{L}$ MTT reagent at a concentration of $5.0 \mathrm{mg} / \mathrm{mL}$ in phosphate-buffered saline (PBS) (Sigma Chemical) for 4 hours at $37^{\circ} \mathrm{C}$, at which point the supernatant was removed and dimethyl sulfoxide was added. Optical density was determined using an automatic microplate reader (Bio-Tek Instruments Inc., Winosski, VT, USA) at a wavelength of $490 \mathrm{~nm}$. The MTT assay was carried out in triplicate to ensure reproducibility.

\section{Flow cytometry}

Cells were detached using $0.2 \%$ trypsin, harvested, and washed with PBS. The cells were then centrifuged at $500 \mathrm{~g}$ 
for $10 \mathrm{~min}$, before the supernatant was discarded. The cells were resuspended in $70 \%$ ethanol for at least 12 hours at $-20{ }^{\circ} \mathrm{C}$ with $10 \mu \mathrm{g} / \mathrm{mL}$ RNase A (Sigma Chemical). Then, the cells were incubated with $1 \mathrm{~mL}$ propidium iodide (Sigma Chemical) for $30 \mathrm{~min}$ at room temperature. Finally, $1 \times 10^{6}$ cells $/ \mathrm{mL}$ were analyzed using the EPICS XL-MCL model counter (Beckman Coulter, Fullerton, CA, USA).

\section{TUNEL assay}

Cells were seeded at a density of $1 \times 10^{5} / \mathrm{mL}$ per coverslip, and then fixed with $4 \%$ paraformaldehyde in PBS for 1 hour. After washing with PBS, the cells were permeabilized with $0.1 \%$ Triton $\mathrm{X}-100$ and $0.1 \%$ sodium citrate for $2 \mathrm{~min}$ at $4{ }^{\circ} \mathrm{C}$. TUNEL staining was performed using the in situ Cell Death Detection Kit Fluorescein (Roche, Mannheim, Germany). The manufacturer's protocol was followed, and a fluorescence microscope (ECLIPSE 80i), digital camera (DXM 1200F) and ACT-1 software (version 2.63, Nikon, Tokyo, Japan) were used to assess cell death.

\section{PGE2 ELISA}

An ELISA was performed to determine the concentration of PGE2 using a PGE2 Parameter Assay Kit according to the manufacturer's instructions (R\&D Systems, Minneapolis, MN, USA). The supernatants collected after treatment of the cells with survivin and paeonol were added to wells coated with anti-PGE2 antibody. After incubation for 1 hour at $37^{\circ} \mathrm{C}$, the wells were washed 4 times with PBS-Tween 20 (pH 7.4) and further incubated for 1 hour with alkaline phosphatase-conjugated anti-PGE2 antibody. The wells were then washed 4 times with PBS-Tween 20 and incubated with substrate solution for 1 hour. The relative absorbance was measured at $450 \mathrm{~nm}$ using a microplate reader (Thermo Fisher) and the concentration was calculated using a standard curve.

\section{Western blotting}

Cells were lysed with RIPA buffer and the protein concentration was determined using the Lowry protein assay. The protein $(40 \mu \mathrm{g})$ was loaded onto $10 \%$ polyacrylamide gels and transferred onto polyvinylidene difluoride membranes (MilliPore, Bedford, MA, USA). Tris-buffered saline Tween-20 containing 5\% nonfat dry milk was used to block the membranes overnight. The membranes were incubated overnight with primary antibodies against survivin $(1: 2,000$; sc- 8807 , Santa Cruz Biotechnology) and COX-2 (1:2,000, sc-1746, Santa Cruz Biotechnology) at $4{ }^{\circ} \mathrm{C}$ and washed 3 times with PBS. Then, the membranes were incubated with a horseradish peroxidase-conjugated secondary antibody (diluted 1:2,000) for 2 hours at room temperature. The Enhanced Chemiluminescence Western Blotting Detection Kit (ECL, Pierce, Rockford, IL, USA) was used for detection. ImageJ was used to calculate the band intensity.

\section{Statistical analysis}

Each assay was repeated at least three times. Pearson's chi-square test and Student's $t$-test were used to compare differences between groups. Data are presented as mean \pm standard deviation. $\mathrm{P}<0.05$ was considered to be significant.

\section{Results}

\section{Survivin and COX-2 expression in HCC tissue}

Immunohistochemical examination of paraffin-embedded HCC specimens $(\mathrm{n}=57)$ detected survivin in the nucleus and/or cytoplasm in 47 of 57 specimens (82.5\%) (Figure $2 A$ ). This suggested that survivin was overexpressed in HCC compared with adjacent normal tissues (Figure $2 B$ ) and might be a key target for anti-HCC treatment strategies. The association between survivin and COX-2 expression was also examined. The expression of COX-2 was analyzed by IHC in the same HCC specimens and adjacent normal tissues (Figure $2 C, D$ ). The results showed that survivin was expressed in $90.2 \%(37 / 41)$ of COX2-positive specimens, while $37.5 \%(6 / 16)$ of the COX-2-negative specimens were survivin-negative (Table 1), suggesting an association between survivin and COX-2.

\section{Regulation of survivin expression in HCC cells by the COX-2/PGE2 pathway}

The possible association between survivin and the COX2/PGE2 synthase pathway was assessed by treating the HCC cells with PGE2 to determine whether it stimulated survivin expression. HepG2 cells and SMMC-7721 cells were treated with PGE2 $(10 \mu \mathrm{M})$ for $0,6,12$, and 24 hours and Western blotting was performed. After treatment with PGE2, survivin levels were found to be significantly increased in HepG2 cells (Figure 2E) and SMMC-7721 cells (Figure $2 F)(\mathrm{P}<0.01$ for both). A graphic presentation 

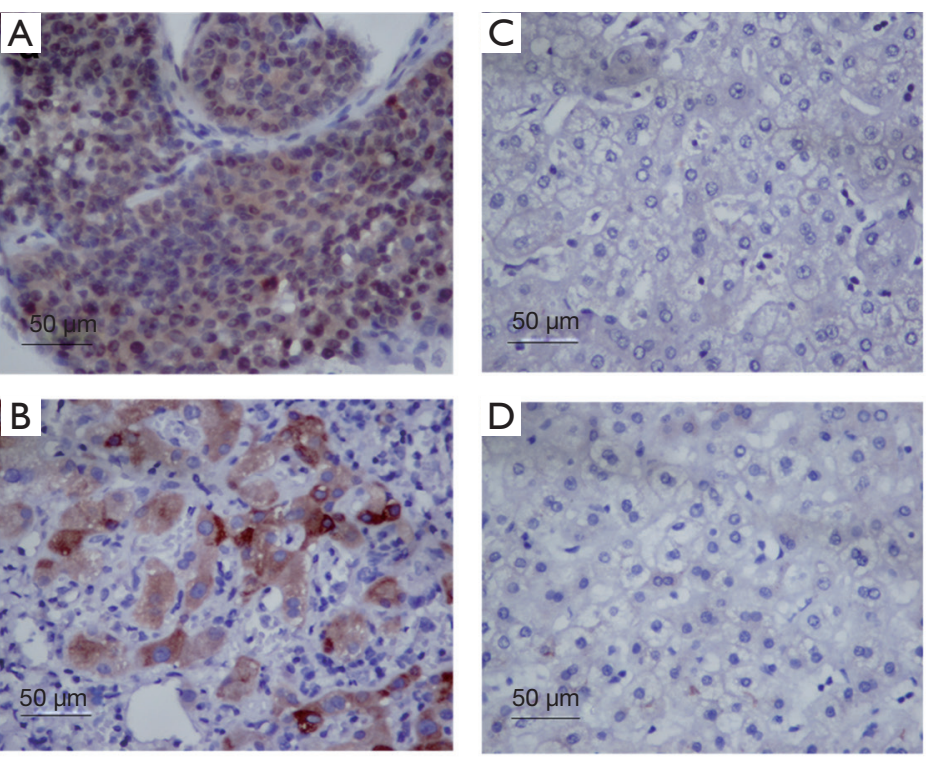

E

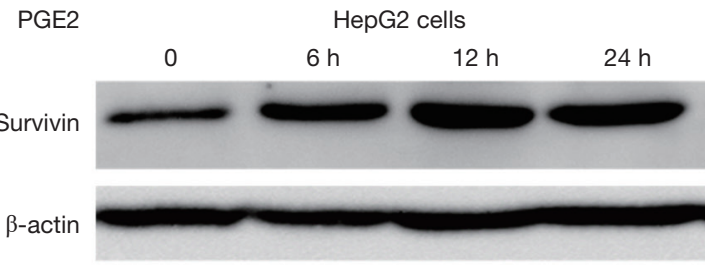

$\mathrm{F}$
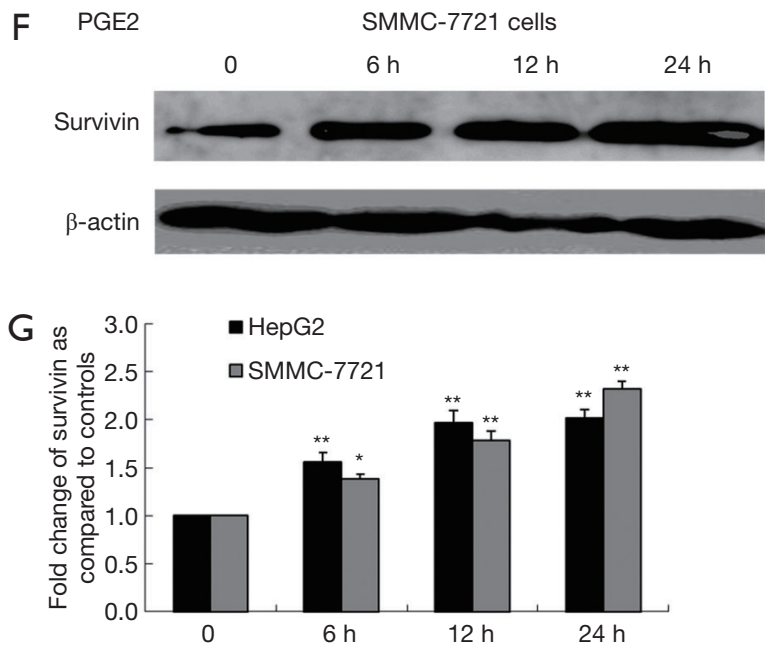

Figure 2 Correlation of the overexpression of survivin in human hepatocellular carcinoma (HCC) specimens with the cyclooxygenase-2/ PGE2 pathway. (A,B,C,D) Overexpression of survivin and cyclooxygenase-2 in HCC and adjacent normal tissues (streptavidin-peroxidase, $\times 400$ ). Western blots showing survivin expression in HepG2 cells (E) and SMMC-7721 cells (F) after treatment with prostaglandin E2 $(10 \mu \mathrm{M})$ for $0,6,12$, and 24 hours as compared with the untreated group, with $\beta$-actin as internal control. (G) Optical density (OD) values of survivin versus $\beta$-actin are presented as fold-change of the control values $\left({ }^{*}, \mathrm{P}<0.05 ;{ }^{* *}, \mathrm{P}<0.01\right)$.

of the results is shown in Figure 2G. The optical density values of survivin versus $\beta$-actin are presented as a foldchange of the control values (Figure $2 G$ ). These findings suggested that the COX-2/PGE2 pathway might be critical for upregulation of survivin in HCC.

\section{Inbibition of cell growth and induction of apoptosis in HCC cells by paeonol}

The effect of paeonol in HCC cells was determined by MTT assay. As the dose of paeonol increased, the viability of treated cells decreased compared with that of untreated cells (HepG2 cells: Figure 3A; SMMC-7721 cells: Figure 3B).

The induction of apoptosis by paeonol was assessed by TUNEL assay using both cell lines. Apoptosis was increased in paeonol-treated cells as compared with untreated cells (Figure 4A: untreated HepG2 cells; Figure 4B: HepG2 cells treated with $94 \mu \mathrm{M}$ paeonol; Figure $4 C$ : HepG2 cells treated
Table 1 Expression of cyclooxygenase 2 (COX-2) and survivin in human hepatocellular carcinoma tissues

\begin{tabular}{lccccc}
\hline \multirow{2}{*}{ Cox-2 } & \multicolumn{3}{c}{ Survivin } & & P value \\
\cline { 2 - 3 } & - & & & \\
\hline Negative & 6 & 10 & & 0.328 & $0.013^{\star}$ \\
Positive & 4 & 37 & & \\
\hline
\end{tabular}

*, $\mathrm{P}<0.05$.

with $188 \mu \mathrm{M}$ paeonol; Figure $4 D$ : HepG2 cells treated with $376 \mu \mathrm{M}$ paeonol; Figure $4 E$ : untreated SMMC-7721 cells; Figure 4F: SMMC-7721 cells treated with $94 \mu \mathrm{M}$ paeonol; Figure 4G: SMMC-7721 cells treated with $188 \mu \mathrm{M}$ paeonol; Figure 4H: SMMC-7721 cells treated with $376 \mu \mathrm{M}$ paeonol). Figure 4I (HepG2 cells) and Figure 47 (SMMC-7721 cells) show the percentage of apoptotic cells treated with different concentrations of paeonol. The percentage of apoptotic cells increased as paeonol concentration increased, with 

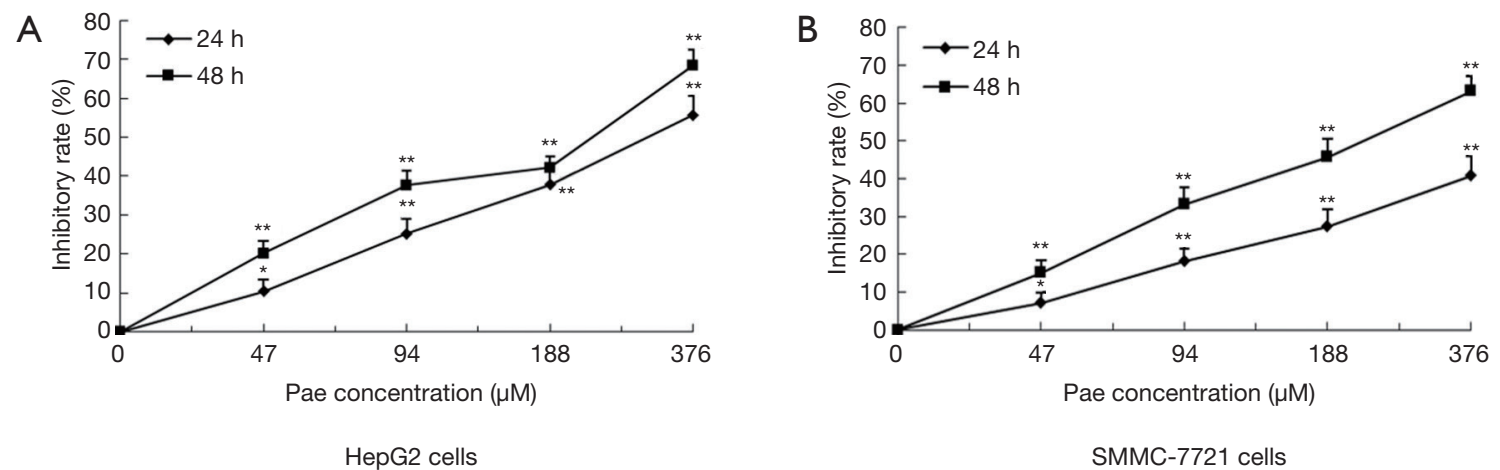

Figure 3 Effect of paeonol (Pae) on cell viability of human hepatocellular carcinoma cells. Graphical representation of the viability of HepG2 (A) and SMMC-7721 (B) cells obtained by MTT assay at different Pae concentrations (47, 94, 188, and 376 $\mu$ M) for 24 and 48 hours. Data obtained in triplicate compared with the untreated groups and shown as mean \pm standard deviation $(\mathrm{SD})$. *, $\mathrm{P}<0.05$, **, $\mathrm{P}<0.01$.

the highest number of apoptotic cells being observed at the maximum concentration of paeonol $(376 \mu \mathrm{M})$ (Figure $4 D, H)$. Furthermore, flow cytometric analysis showed that paeonol increased the sub-G1 population of HepG2 and SMMC7721 cells in a dose-dependent manner (Figure $5 A$ : untreated HepG2 cells; Figure 5B: HepG2 cells treated with $94 \mu \mathrm{M}$ paeonol; Figure 5C: HepG2 cells treated with $188 \mu \mathrm{M}$ paeonol; Figure 5D: HepG2 cells treated with $376 \mu \mathrm{M}$ paeonol; Figure 5E: untreated SMMC-7721 cells; Figure 5F: SMMC-7721 cells treated with $94 \mu \mathrm{M}$ paeonol; Figure 5G: SMMC-7721 cells treated with $188 \mu \mathrm{M}$ paeonol; Figure $5 H$ : SMMC-7721 cells treated with $376 \mu M$ paeonol).

\section{Effect of paeonol on the expression of survivin}

The effect of paeonol treatment on survivin expression was evaluated by Western blotting. HepG2 and SMMC-7721 cells treated with paeonol for 24 hours showed a decrease in the level of survivin as compared with cells without paeonol treatment (Figure 6A,B,C,D). The expression of both COX2 and survivin decreased gradually as the concentration of paeonol increased. The maximum reduction in the protein levels was seen at the maximum concentration of paeonol $(376 \mu M)$ (Figure 6E,F). Therefore, the paeonol-induced alteration in the level of survivin might contribute to the susceptibility of HCC cells to paeonol-induced apoptosis.

\section{Effect of paeonol on the COX-2/PGE2 patbway and survivin in HCC cells}

The role played by the COX-2/PGE2 pathway in paeonol- mediated survivin degradation in HCC cells was elucidated by assessing the expression levels of COX-2 (Figure 6A,B,C,D) and PGE-2 (Figure 6E, $F$ ) in paeonol-treated cells using Western blotting and ELISA, respectively. Paeonol significantly inhibited COX-2 expression and decreased PGE2 levels in HCC cells (Figure 6). Further, PGE2 treatment alone resulted in increased levels of survivin, but combined treatment with PGE2 and paeonol decreased survivin expression (Figure 7A: HepG2 cells; Figure 7B: SMMC-7721 cells). The relative expression levels of both treatment with PGE2 alone and combined treatment with PGE2 and paeonol are shown in Figure 7C. Moreover, Western blotting showed that treatment with the COX-2 specific inhibitor, NS-398 downregulated survivin and COX-2 expression (Figure 8A,B,C,D; Figure 8A,C show HepG2 cells, Figure $8 B, D$ show SMMC-7721 cells), and downregulated PGE2 levels were demonstrated by ELISA (Figure 8E). Compare with untreated HepG2 cells (Figure 9A), the sub-G1 fraction of HepG2 cells was found to be increased by treatment with NS-398 (Figure 9B) and by paeonol treatment (Figure 9C) by flow cytometry, which was also observed in SMMC-7721 cells (Figure 9D,E,F). This was further confirmed by TUNEL staining. Compared with untreated HepG2 cells (Figure 9G), the proportion of TUNEL-positive cells treated with NS398 (Figure 9H) and paeonol (Figure 9I) was increased, which was also observed in SMMC-7721 cells (Figure 97,K,L). The percentage of apoptotic cells in both cell lines compared with untreated cells was shown in Figure 9M. Taken together, these findings suggested that the inhibition of survivin by paeonol was regulated by the COX-2/PGE2 pathway. 

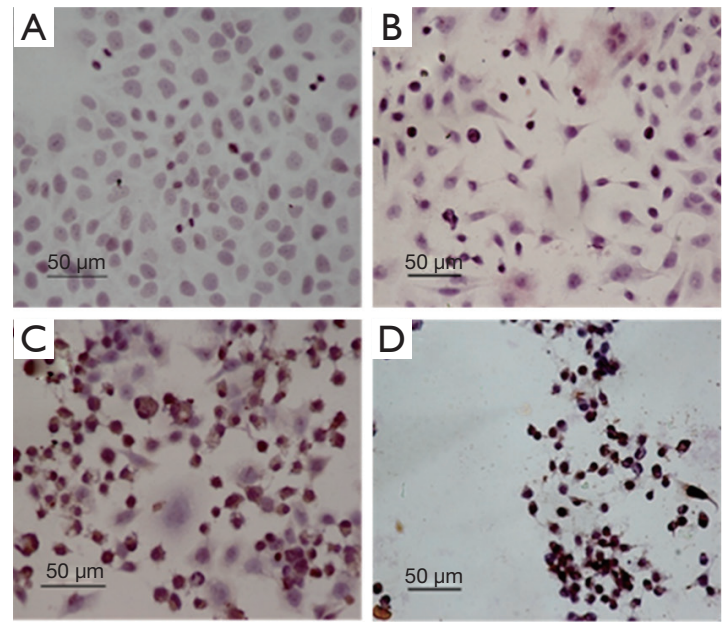

I

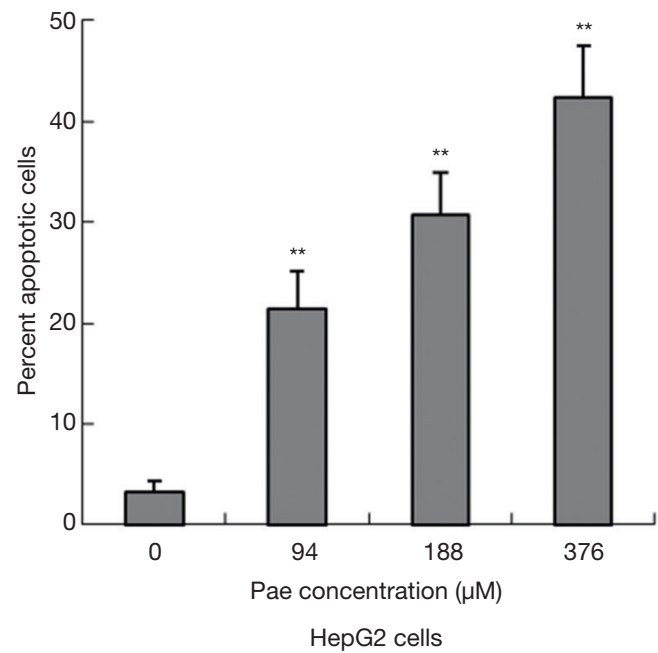

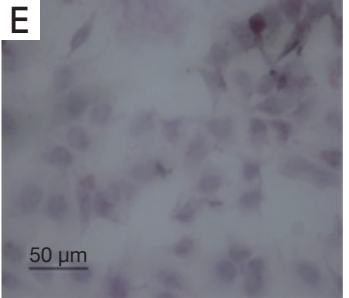
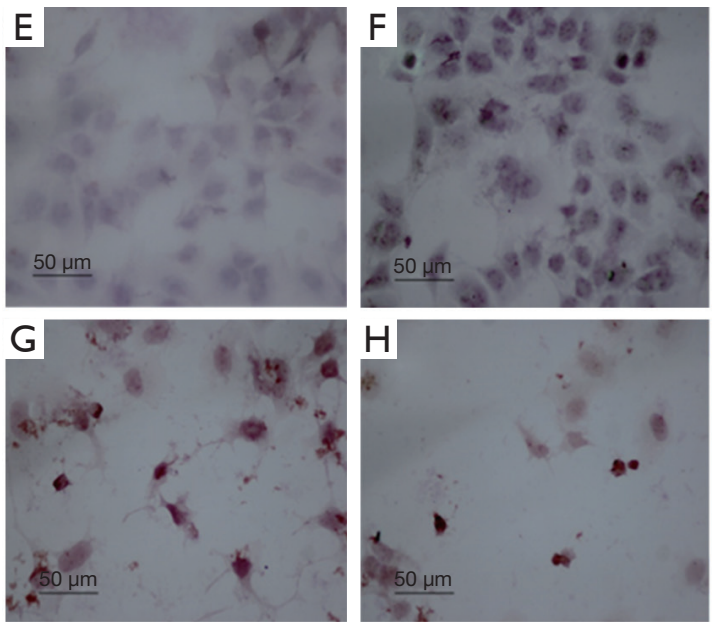

J

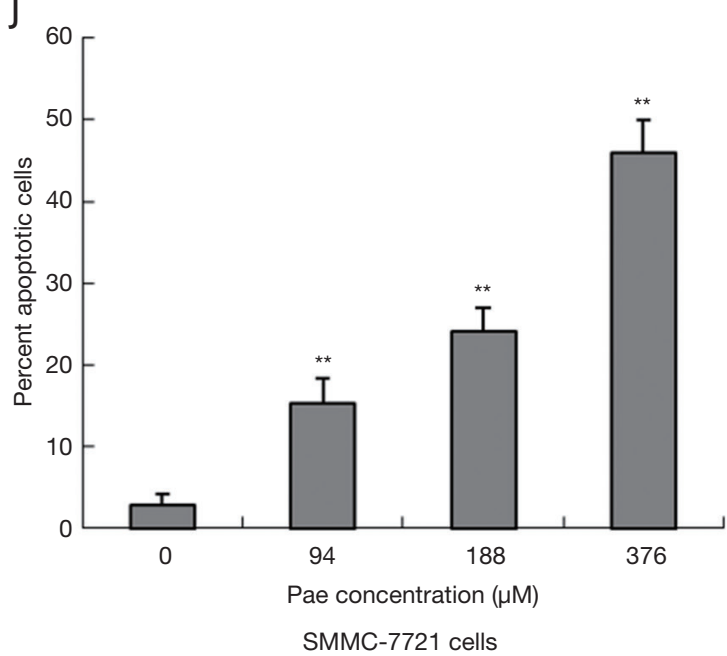

Figure 4 Terminal deoxynucleotidyl transferase dUTP nick end labeling (TUNEL) assay showing paeonol-induced apoptosis in human hepatocellular carcinoma cells. Morphology and percentage of apoptotic cells assessed by TUNEL staining. Cells with brown nuclei were defined as apoptotic. Hepatocellular carcinoma cells treated with various concentrations of paeonol (Pae: 94, 188, or $376 \mu M$ ) [left (A,B,C,D,I): HepG2 cells; right (E,F,G,H,J): SMMC-7721 cells] for 24 hours. (A) Untreated HepG2 cells; (B) HepG2 cells treated with $94 \mu M$ Pae; (C) HepG2 cells treated with $188 \mu M$ Pae; (D) HepG2 cells treated with $376 \mu M$ Pae; (E) untreated SMMC-7721 cells; (F) SMMC-7721 cells treated with $94 \mu M$ Pae; (G) SMMC-7721 cells treated with $188 \mu M$ Pae; (H) SMMC-7721 cells treated with $376 \mu M$ Pae. (I,J) Percentage of apoptotic cells treated with different concentrations of paeonol: (I) HepG2 cells and (J) SMMC-7721 cells. Data are presented as mean \pm standard deviation. ${ }^{* *}, \mathrm{P}<0.01$.

\section{Discussion}

Identification of the signaling pathways involved in HCC pathogenesis and elucidation of their effects, functions, and interactions would help to improve HCC treatment. We found that $82.5 \%$ of the human HCC specimens collected in the present study were positive for survivin. Furthermore, the expression of COX-2 was positively related to that of survivin, consistent with the findings of a previous study (25). Moreover, PGE2, the main product of COX-2, promoted survivin expression in HepG2 cells and SMMC-7721 cells, indicating that the COX-2/PGE2 pathway plays a critical role in survivin regulation in HCC, which has not been reported before.

The results of the present study showed that paeonol significantly enhanced apoptosis and increased the sub-G1 

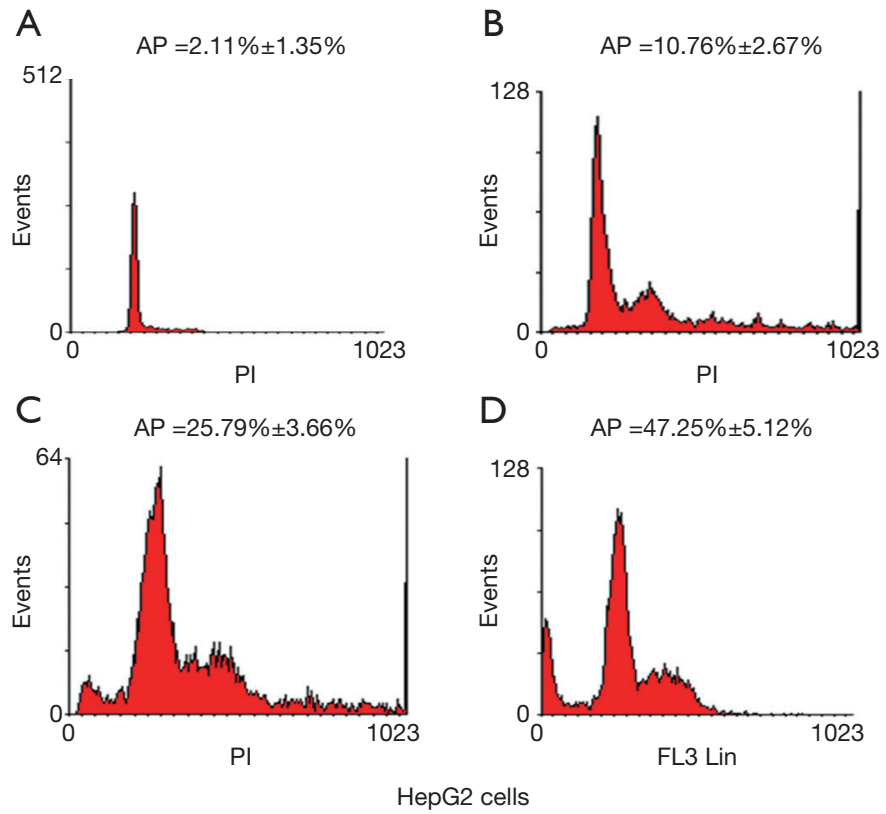

B

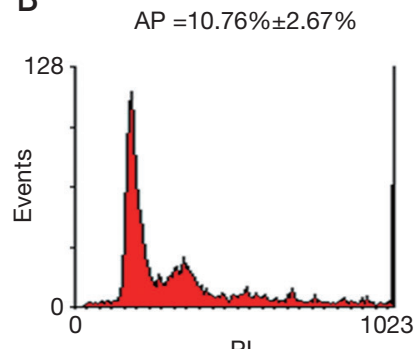

$E$
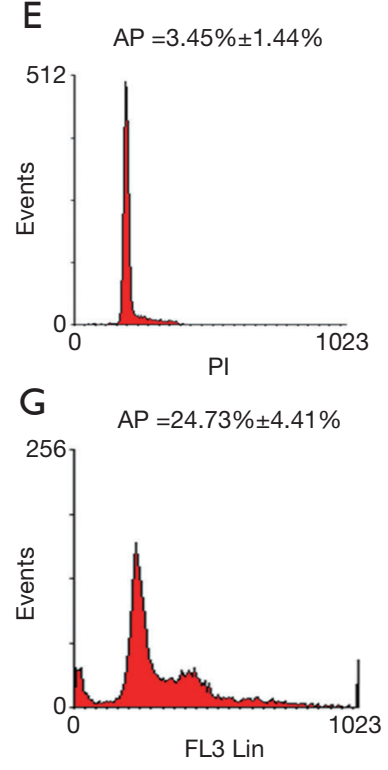

$\mathrm{F}$

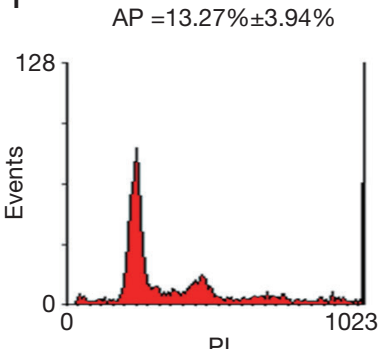

$\mathrm{H} \quad \mathrm{AP}=50.23 \% \pm 4.82 \%$

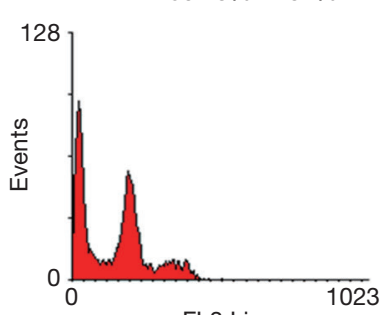

SMMC-7721 cells

Figure 5 Flow cytometric analysis of paeonol-induced apoptosis in human hepatocellular carcinoma (HCC) cells. HCC cells were treated with various concentrations of paeonol (Pae: 94, 188, or $376 \mu \mathrm{M}$ ) [left (A,B,C,D): HepG2 cells; right (E,F,G,H): SMMC-7721 cells] for 24 hours. Fluorescence-activated cell sorting (FACS) analysis indicated the sub-G1 proportion: (A) untreated HepG2 cells; (B) HepG2 cells treated with $94 \mu$ M Pae; (C) HepG2 cells treated with $188 \mu$ M Pae; (D) HepG2 cells treated with $376 \mu$ M Pae; (E) untreated SMMC-7721 cells; (F) SMMC-7721 cells treated with $94 \mu M$ Pae; (G) SMMC-7721 cells treated with $188 \mu M$ Pae; (H) SMMC-7721 cells treated with $376 \mu M$ Pae. Data are presented as mean \pm standard deviation. **, $\mathrm{P}<0.01$.

fraction of HCC cells, a finding consistent with that of previous studies $(11,26)$. The proliferation of HCC cells was also suppressed by paeonol, which confirmed a previous report that paeonol might induce HCC cell apoptosis via the NF- $\kappa \mathrm{B}$ pathway (11). However, the effect of paeonol on survivin in HCC has not been previously examined. Survivin is thought to play an essential role in tumorigenesis, and the possibility of exploiting it as a therapeutic target has been suggested (18). Therefore, to facilitate the identification of new, potent antitumor drugs for HCC patients, it is crucial that the mechanisms of survivin regulation are elucidated. We showed that paeonol has a role in regulating survivin in HepG2 and SMMC-7721 cells; survivin levels were significantly reduced after the cells were treated with paeonol, a phenomenon that has been previously observed in ovarian cancer cells (7). Our results, therefore, suggested that paeonol might suppress the tumorigenicity of human HCC cells by inhibiting survivin expression, and based on this, the expression of COX-2 after treatment with paeonol was assessed. We found that paeonol downregulated the levels of COX-2 in HepG2 and SMMC-7721 cells, thereby decreasing the levels of PGE2. Paeonol has previously been reported to reduce the expression levels of survivin and COX-2 in lung adenocarcinoma cells (27), as well as the expression of survivin in ovarian cancer cells (7). A previous study revealed that meloxicam, a nonsteroidal anti-inflammatory drug, targeted survivin, via a COX-2dependent pathway, to induce apoptosis in HCC cells (28). The findings of the present study suggest that survivin might be inhibited by paeonol via a reduction in the level of COX-2. As previously reported in HUH-7 cells, another HCC cell line, treatment with PGE2 increased survivin expression significantly in both cell lines (22). Interestingly, this effect was reversed by a combination treatment of paeonol and PGE2, suggesting that paeonol might regulate survivin via PGE2. This supposition was further confirmed when HepG2 and SMMC-7721 cells were treated with a potent COX-2 inhibitor, NS-398, and the expression levels of COX-2 and PGE2 as well as those of survivin were reduced, leading to induction of apoptosis in HepG2 and 


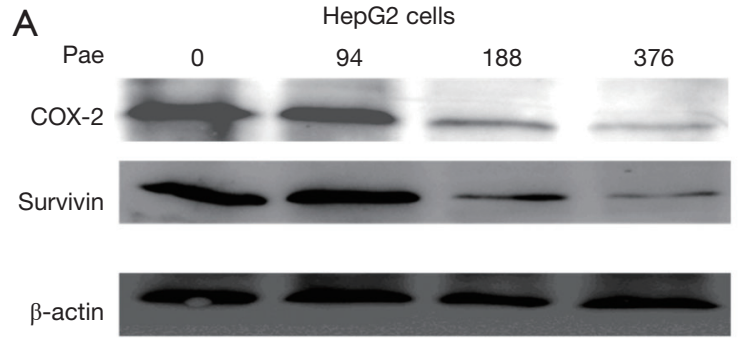

C

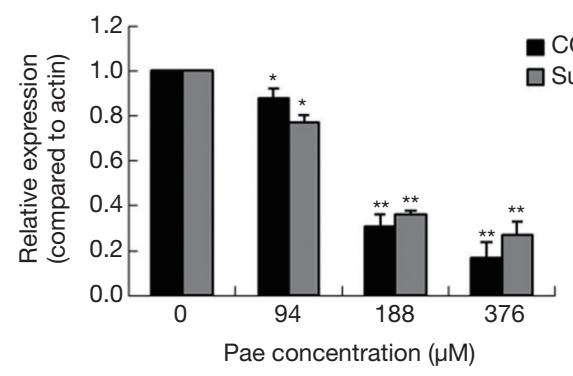

E

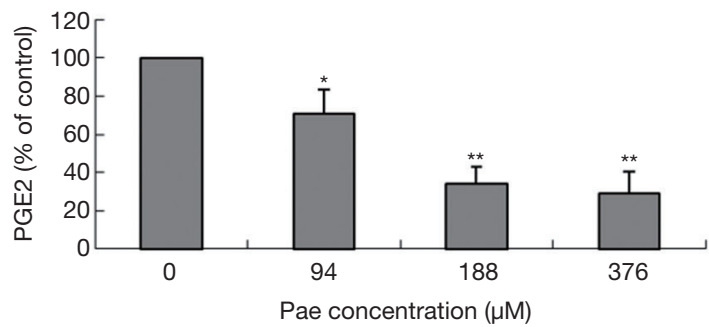

B
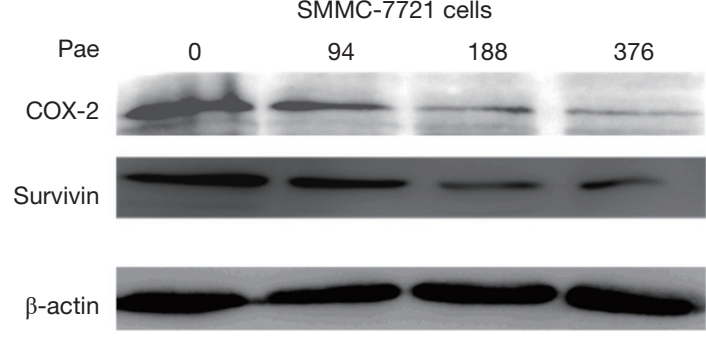

D

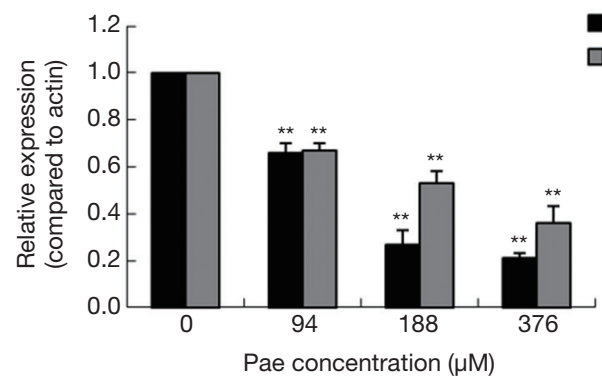

F

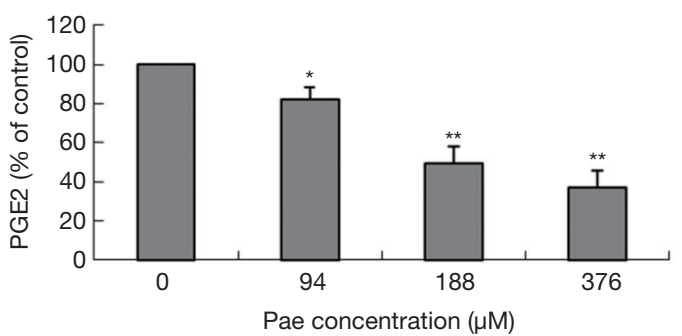

Figure 6 Effects of paeonol (Pae) on cyclooxygenase-2 (COX-2), survivin, and prostaglandin E2 (PGE2) in human hepatocellular carcinoma cells. Cells were exposed to Pae at various concentrations $(94,188$, and $376 \mu \mathrm{M})$ for 24 hours. Western blots and graphical representations show the expression levels of survivin and COX-2 in the cell lysates: (A,C) HepG2 cells; (B,D) SMMC-7721 cells, with $\beta$-actin as internal reference. Optical density (OD) values of survivin and COX-2 versus $\beta$-actin are represented as fold change of the control values $\left({ }^{*}\right.$, $\mathrm{P}<0.05$; **, $\mathrm{P}<0.01)$. (E,F) Graphical representation of ELISA results obtained at various Pae concentrations in HepG2 cells (E), and SMMC-7721 cells $(\mathrm{F})$. Data are presented as mean \pm standard deviation (bars $=\mathrm{SD}$ ) **, $\mathrm{P}<0.01$. ELISA, enzyme-linked immunosorbent assay; SD, standard deviation.
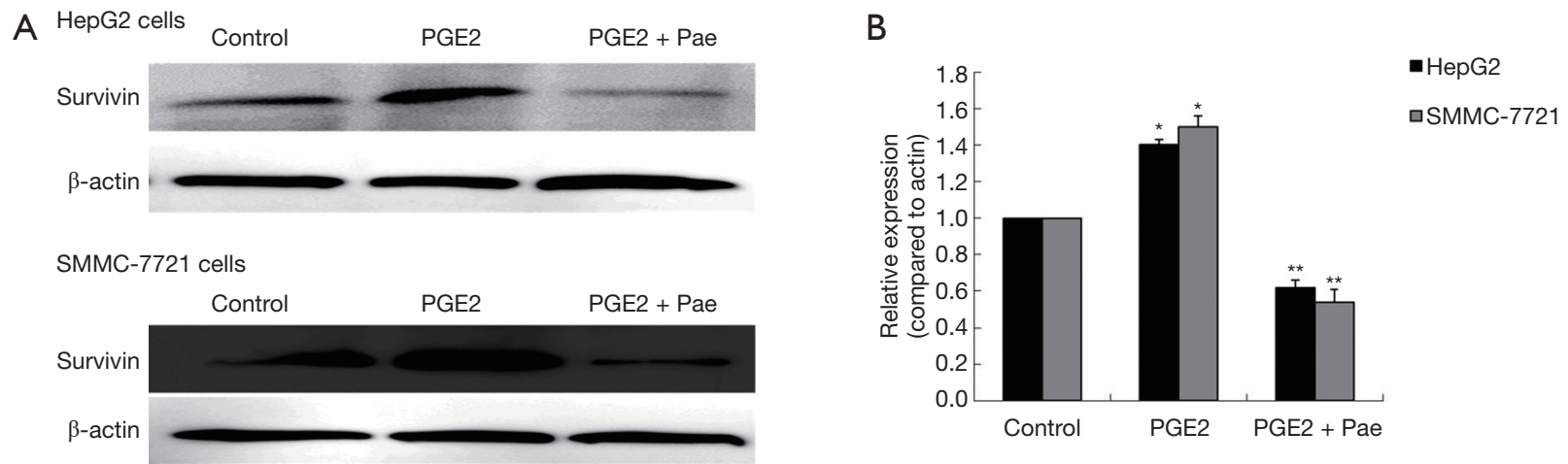

Figure 7 Effect of combined treatment with paeonol (Pae) and prostaglandin E2 (PGE2) on survivin in human hepatocellular carcinoma cells. (A,B) Western blots obtained after exposure of cells to PGE2 $(10 \mu \mathrm{M})$ in the absence or presence of Pae $(376 \mu \mathrm{M})$ for 24 hours with $\beta$-actin as internal reference: (A) HepG2 cells; (B) SMMC-7721 cells. (C) Optical density (OD) values versus $\beta$-actin presented as fold change of the control values $\left({ }^{*}, \mathrm{P}<0.05 ;{ }^{* *}, \mathrm{P}<0.01\right.$, compared with untreated cells). 
A

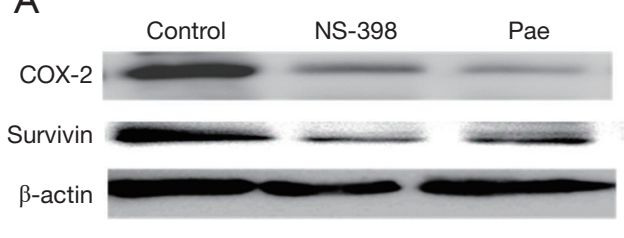

C

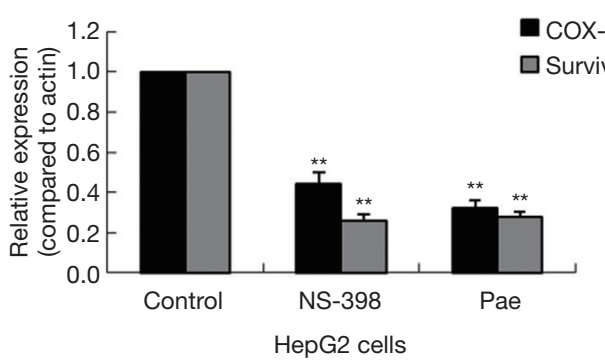

B

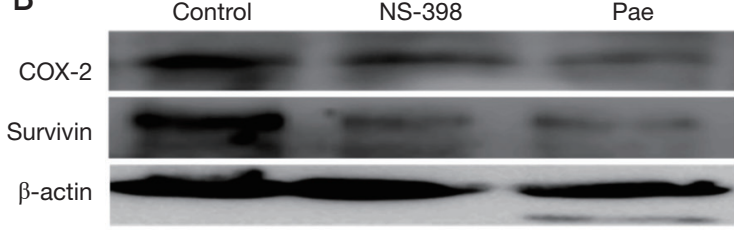

$\mathrm{D}$

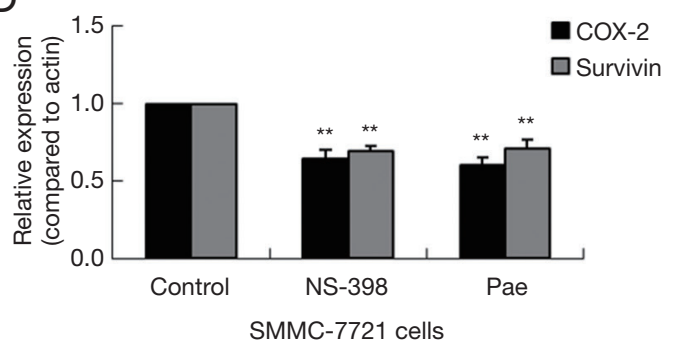

$\mathrm{E}$

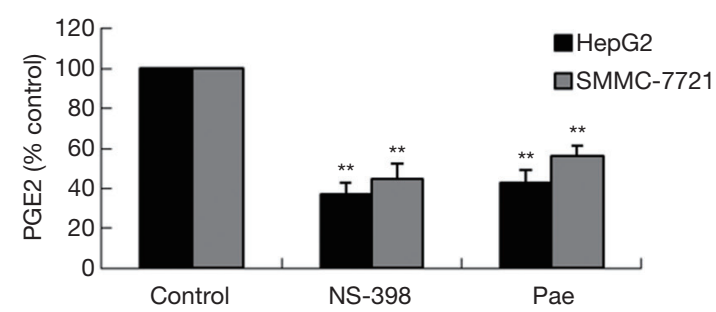

Figure 8 Determination of the effect of NS-398 on cell apoptosis and expression levels of cyclooxygenase-2 (COX-2) and survivin in human hepatocellular carcinoma (HCC) cells by Western blotting. (A,B,C,D) Western blots and graphical representations demonstrating COX2 and survivin expression levels in HCC cells treated with NS-398 and paeonol (Pae), with $\beta$-actin as internal control. Data are presented as mean \pm standard deviation $(\mathrm{SD})\left({ }^{* *}, \mathrm{P}<0.01\right)$. Optical density $(\mathrm{OD})$ values versus $\beta$-actin presented as fold change of the control values $\left(^{*}\right.$, $\mathrm{P}<0.05$; ** $\mathrm{P}<0.01$ ). (E) Concentration of prostaglandin E2 (PGE2) in treated cells compared with untreated cells measured by ELISA. Data are presented as mean $\pm \mathrm{SD}$ of three independent experiments (bars $=\mathrm{SD})\left({ }^{* *}, \mathrm{P}<0.01\right)$. ELISA, enzyme-linked immunosorbent assay.

SMMC-7721 cells. In contrast, treatment with PGE2 alone significantly increased COX-2 expression; however, this was decreased by the treatment of cells with paeonol. These findings, therefore, suggest that the suppression of survivin expression by paeonol is regulated by the COX-2/PGE2 pathway.

The limitations of this study include the lack of in vivo results and the modest sample size. Further mechanismbased in vivo studies and larger patient cohorts are needed to establish the importance of paeonol and to facilitate its development as a pharmacologically safe, non-toxic agent for the treatment of HCC, to be used either alone or in combination with other phytochemical drugs.

\section{Conclusions}

This is the first study, to the best of our knowledge, to demonstrate that paeonol can exert antitumor effects by targeting survivin via the COX-2/PGE2 signaling pathway in HCC. Further in vivo studies are needed to establish the importance of paeonol in cancer treatment. Through inhibiting cell proliferation and inducing apoptosis, paeonol could serve as a promising therapeutic agent in HCC. Elucidation of this novel mechanism may help in the development of new alternate therapeutic options that are safe and applicable for clinical use in the management and treatment of HCC. 

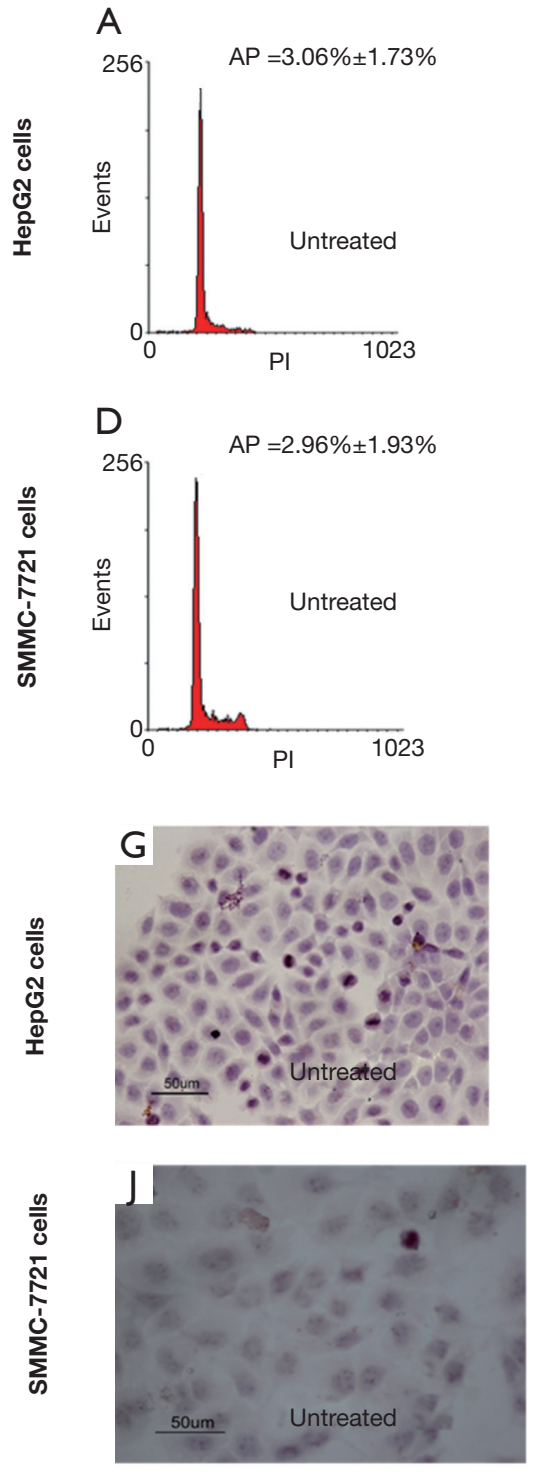
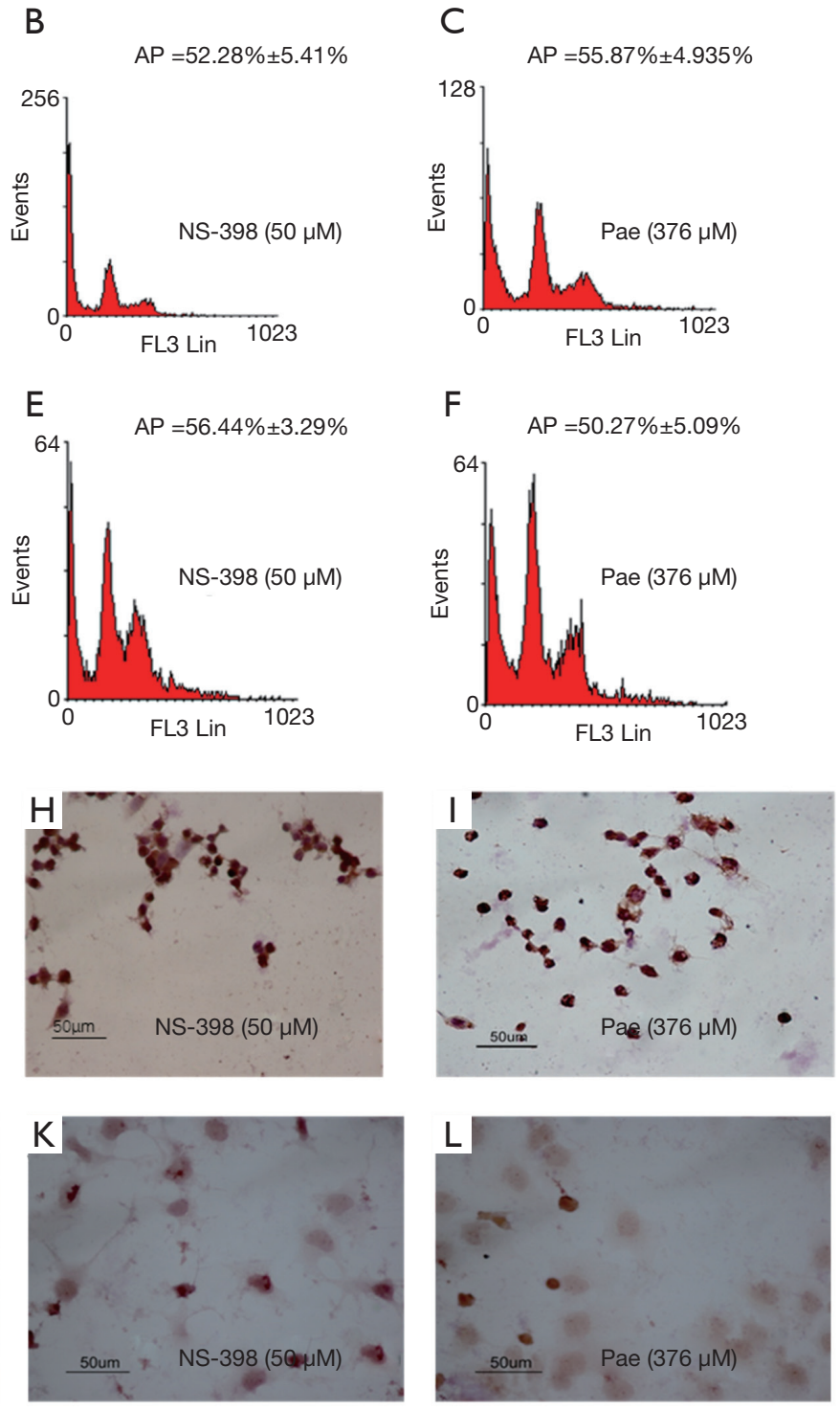

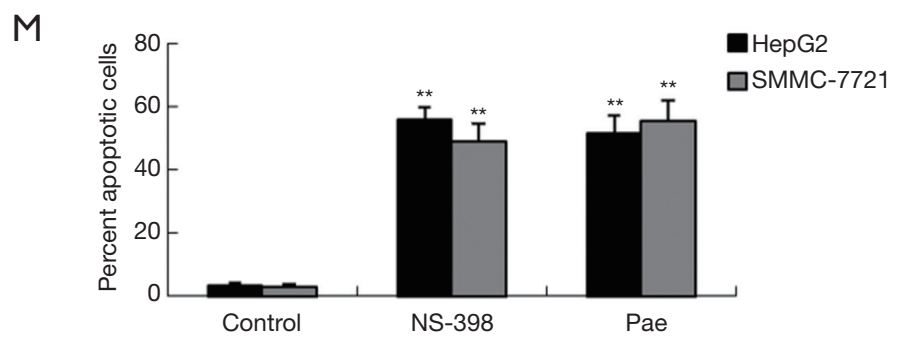

Figure 9 Determination of the effect of NS-398 on cell apoptosis and expression levels of cyclooxygenase-2, survivin, and prostaglandin E2 in human hepatocellular carcinoma (HCC) cells by flow cytometry and TUNEL assay. NS-398 (50 $\mu M$ ) and paeonol (Pae: $376 \mu M)$ were used to treat HCC cells for 24 hours [left (A,B,C,G,H,I): HepG2 cells; right (D,E,F,J,K,L): SMMC-7721 cells] separately. (A,B,C,D,E,F) Sub-G1 analysis by FACS. (G,H,IJ,K,L,M) Morphology and percentage of apoptotic cells by TUNEL staining. Cells with brownstained nuclei were defined as apoptotic. [A,D,G,J: untreated cells; B,E,H,K: NS-398 $(50 \mu \mathrm{M})$ treated cells; C,F,I,L: Pae $(376 \mu \mathrm{M})$ treated cells]. Data are presented as mean \pm standard deviation $(\mathrm{SD})\left({ }^{* *}, \mathrm{P}<0.01\right)$. FACS, fluorescence-activated cell sorting; TUNEL, terminal deoxynucleotidyl transferase dUTP nick end labeling. 


\section{Acknowledgments}

Funding: This work was supported by the Training Program Foundation of Youth Scholars of the First Affiliated Hospital of Anhui Medical University (2017KJ29).

\section{Footnote}

Reporting Checklist: The authors have completed the MDAR checklist. Available at http://dx.doi.org/10.21037/tcr-20$322 \mathrm{~A}$

Data Sharing Statement: Available at http://dx.doi. org/10.21037/tcr-20-322A

Peer Review File: Available at http://dx.doi.org/10.21037/tcr20-322A

Conflicts of Interest: Both authors have completed the ICMJE uniform disclosure form (available at http://dx.doi. org/10.21037/tcr-20-322A). The authors have no conflicts of interest to declare.

Ethical Statement: The authors are accountable for all aspects of the work, ensuring that questions related to the accuracy or integrity of any part of the work were appropriately investigated and resolved. The study was conducted in accordance with the Declaration of Helsinki (as revised in 2013). The protocol was approved by the institutional review board of Anhui Medical University (approval no. Quick-PJ 2019-14-21), and informed consent was given by all patients enrolled.

Open Access Statement: This is an Open Access article distributed in accordance with the Creative Commons Attribution-NonCommercial-NoDerivs 4.0 International License (CC BY-NC-ND 4.0), which permits the noncommercial replication and distribution of the article with the strict proviso that no changes or edits are made and the original work is properly cited (including links to both the formal publication through the relevant DOI and the license). See: https://creativecommons.org/licenses/by-nc-nd/4.0/.

\section{References}

1. Yang JD, Hainaut P, Gores GJ, et al. A global view of hepatocellular carcinoma: trends, risk, prevention and management. Nat Rev Gastroenterol Hepatol
2019;16:589-604.

2. Zhu RX, Seto WK, Lai CL, et al. Epidemiology of hepatocellular carcinoma in the Asia-Pacific region. Gut Liver 2016;10:332-9.

3. Greten TF, Lai CW, Li G, et al. Targeted and immune-based therapies for hepatocellular carcinoma. Gastroenterology 2019;156:510-24.

4. Kudo M, Finn RS, Qin S, et al. Lenvatinib versus sorafenib in first-line treatment of patients with unresectable hepatocellular carcinoma: a randomised phase 3 noninferiority trial. Lancet 2018;391:1163-73.

5. Jemal A, Ward EM, Johnson CJ, et al. Annual report to the nation on the status of cancer, 1975-2014, featuring survival. J Natl Cancer Inst 2017;109:djx030.

6. Zhang L, Li DC, Liu LF. Paeonol: pharmacological effects and mechanisms of action. Int Immunopharmacol 2019;72:413-21.

7. Yin $\mathrm{J}, \mathrm{Wu} \mathrm{N}$, Zeng F, et al. Paeonol induces apoptosis in human ovarian cancer cells. Acta Histochem 2013;115:835-9.

8. Xu Y, Zhu JY, Lei ZM, et al. Anti-proliferative effects of paeonol on human prostate cancer cell lines DU145 and PC-3. J Physiol Biochem 2017;73:157-65.

9. Saahene RO, Wang J, Wang ML, et al. The antitumor mechanism of paeonol on CXCL4/CXCR3-B signals in breast cancer through induction of tumor cell apoptosis. Cancer Biother Radiopharm 2018;33:233-40.

10. Fu J, Yu L, Luo J, et al. Paeonol induces the apoptosis of the SGC-7901 gastric cancer cell line by downregulating ERBB2 and inhibiting the NF- $\kappa B$ signaling pathway. Int J Mol Med 2018;42:1473-83.

11. Li Q, Zhang Y, Sun J, et al. Paeonol-mediated apoptosis of

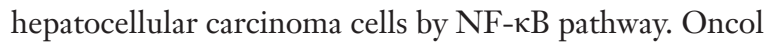
Lett 2019;17:1761-7.

12. Chen B, Ning M, Yang G. Effect of paeonol on antioxidant and immune regulatory activity in hepatocellular carcinoma rats. Molecules 2012;17:4672-83.

13. Fan L, Song B, Sun G, et al. Endoplasmic reticulum stressinduced resistance to doxorubicin is reversed by paeonol treatment in human hepatocellular carcinoma cells. PLoS One 2013;8:e62627.

14. Shintani M, Sangawa A, Yamao N, et al. Smac/DIABLO expression in human gastrointestinal carcinoma: Association with clinicopathological parameters and survivin expression. Oncol Lett 2014;8:2581-6.

15. Shi CT, Ma J, Shi QF, et al. High survivin and low zinc finger of the cerebellum 1 expression indicates poor prognosis in triple-negative breast carcinoma. J Breast 
Cancer 2019;22:248-59.

16. Sumi T, Hirai S, Yamaguchi M, et al. Survivin knockdown induces senescence in TTF1-expressing, KRAS-mutant lung adenocarcinomas. Int J Oncol 2018;53:33-46.

17. Fernández JG, Rodriguez DA, Valenzuela $M$, et al. Survivin expression promotes VEGF-induced tumor angiogenesis via PI3K/Akt enhanced beta-catenin/Tcf-Lef dependent transcription. Mol Cancer 2014;13:209.

18. Martínez-García D, Manero-Ruperez N, Quesada R, et al. Therapeutic strategies involving survivin inhibition in cancer. Med Res Rev 2019;39:887-909.

19. Chen Y, Chen HN, Wang K, et al. Ketoconazole exacerbates mitophagy to induce apoptosis by downregulating cyclooxygenase-2 in hepatocellular carcinoma. J Hepatol 2019;70:66-77.

20. Zhang YS, Shen Q, Li J. Traditional Chinese medicine targeting apoptotic mechanisms for esophageal cancer therapy. Acta Pharmacol Sin 2016;37:295-302.

21. Nagtegaal ID, Odze RD, Klimstra D, et al. The 2019 WHO classification of tumours of the digestive system. Histopathology 2020;76:182-8.

22. Bai XM, Jiang H, Ding JX, et al. Prostaglandin E2 upregulates survivin expression via the EP1 receptor in hepatocellular carcinoma cells. Life Sci 2010;86:214-23.

Cite this article as: Liu H, Zhang C. Paeonol induces antitumor effects in hepatocellular carcinoma cells through survivin via the cyclooxygenase-2/prostaglandin E2 signaling pathway. Transl Cancer Res 2020;9(11):7183-7195. doi: $10.21037 /$ tcr-20-322A
23. Xu SP, Sun GP, Shen YX, et al. Synergistic effect of combining paeonol and cisplatin on apoptotic induction of human hepatoma cell lines. Acta Pharmacol Sin 2007;28:869-78.

24. $\mathrm{Hu} \mathrm{KQ}$, Yu CH, Mineyama Y, et al. Inhibited proliferation of cyclooxygenase-2 expressing human hepatoma cells by NS-398, a selective COX-2 inhibitor. Int J Oncol 2003;22:757-63.

25. Yang Y, Zhu J, Gou H, et al. Clinical significance of Cox-2, Survivin and Bcl-2 expression in hepatocellular carcinoma (HCC). Med Oncol 2011;28:796-803.

26. Chunhu Z, Suiyu H, Meiqun C, et al. Antiproliferative and apoptotic effects of paeonol on human hepatocellular carcinoma cells. Anticancer Drugs 2008;19:401-9.

27. Lei Y, Li HX, Jin WS, et al. The radiosensitizing effect of paeonol on lung adenocarcinoma by augmentation of radiation-induced apoptosis and inhibition of the PI3K/ Akt pathway. Int J Radiat Biol 2013;89:1079-86.

28. Dong X, Li R, Xiu P, et al. Meloxicam executes its antitumor effects against hepatocellular carcinoma in COX-2- dependent and -independent pathways. PLoS One 2014;9:e92864.

(English language editors: K. Brown and J. Reynolds) 